\section{Sexually dimorphic behavior genes}

Sex hormones, such as estrogen and testosterone, regulate sex-specific behaviors, but very few of the downstream genes carrying out the effects of sex hormones are known. Now, Nirao Shah and colleagues report an unbiased screen for genes with sexually dimorphic expression in the mouse hypothalamus and amygdala, validating 16 genes as having such an expression pattern through the use of microarrays and ISH (Cell 148, 596-607, 2012). The authors performed ISH in castrated male mice, where they observed changes in gene expression consistent with a regulatory role for testicular hormones, and reintroduction of testosterone into castrated mice restored the normal expression patterns of most genes. Mice with knockout alleles of four dimorphic genes were analyzed for a range of sex-typical behaviors in males and females. Males lacking Syt/4 sniffed females less but intromitted (penetrated) in more assays, while females lacking Irs 4 or Cckar showed deficits in female-specific behaviors. Irs4-null mothers took longer to retrieve pups that wandered from the nest and also showed deficits in maternal aggression, attacking intruders in the cage less often than control mothers did. Cckar-null females allowed fewer intromissions from approaching males and were less receptive than control mice. These results show that aspects of sex-specific behavior are regulated by separable genetic pathways.

\section{Acquired resistance in lung cancer}

ALK rearrangements occur in $4 \%$ of non small cell lung cancer patients, which is approximately 8,000 cases in the US and 40,000 cases worldwide each year. Although most $A L K$-positive individuals initially respond to ALK tyrosine kinase inhibitors (TKIs), the majority develop drug resistance and relapse within 1 year. Jeff Engelman and colleagues now report secondary mutations in $A L K$ in individuals with acquired resistance to the ALK TKI crizotinib (Xalkori, Pfizer) (Sci. Transl. Med., published online 25 January 2012; doi:10.1126/scitranslmed.3003316). The authors analyzed tumors from 18 crizotinib-resistant individuals and found that 4 harbored mutations in $A L K$, including 3 missense variants and 1 insertion, with all the mutations affecting amino acids near the ATP-binding domain of ALK. By examining tumor specimens taken before crizotinib administration, the authors confirmed that the $A L K$ mutations were not present before treatment. Each of the $A L K$ variants was expressed in $\mathrm{Ba} / \mathrm{F} 3$ cells that express $E M L 4-A L K$, and their effects on cell survival after crizotinib treatment were tested. All four mutations were found to confer resistance to crizotinib, although to varying degrees. The authors also tested the effect of three next-generation ALK inhibitors and found that each had different levels of activity against particular mutations. The study suggests that personalized combinatorial therapies in crizotinibresistant patients are warranted.

\section{The persistence of founders}

An ongoing goal in evolutionary genetics is to determine the contribution of genetic drift to phenotypic diversity. While there is little doubt that natural selection results in morphological adaptation, evidence for founder effects and population bottlenecks is based mainly on theoretical inference or laboratory simulation. In 2004, a hurricane wiped out

Written by Orli Bahcall, Pamela Feliciano, Wayne Peng \& Kyle Vogan the brown anole (Anolis sagrei) lizard population on several islands in the Bahamas. Jason Kolbe and colleagues seeded seven small islands with randomly selected founding pairs of $A$. sagrei from a nearby large island. They observed subsequent morphological adaptation and genetic variation at six microsatellite loci in the island populations from 2005 to 2009 and report the analysis in a recent paper (Science, published online 2 February 2012; doi:10.1126/science.1209566). Apparent adaption of the length of the hind limb to environmental factors, such as narrower perch and smaller area of vegetation, suggests substantial natural selection. Principle-coordinate analysis (PCoA) of microsatellite markers and ranking tests of hind-limb morphology suggest founder effects, which, surprisingly, persisted throughout the course of the study. This unusual field experiment illustrates the importance of considering founder effects in population genetics studies.

WP

\section{Rare variant association}

With the increasing availability of large-scale sequencing data sets, there has been a growing need for new statistical methods for rare variant association analyses. Several new methods have been described that employ collapsing, weighting and distribution-based approaches. In a recent report, Brent Richards and colleagues evaluate a selection of rare variant association methods, comparing the performance of the methods on a data set of Sanger sequencing results for seven genes in 1,998 individuals (PLoS Genet. 8, e1002496, 2012). The authors also develop a new rare variant association method for continuous traits called weighted outlier detection (WOD), which is based on the KBAC method. They test each method using simulations under a range of scenarios that reflect assumptions on disease architecture and association, finding that these parameters greatly influence the performance of each method, and they note under which scenarios each method performs best. In additional simulations, they examine performance across ranges of power, effect size and proportion of causal variants for both continuous and dichotomous traits. They recommend that, as the power for all the tested methods remains low, studies should include sensitivity analyses and comparisons using several different methods, in addition to replication of results in an independent sample.

$O B$

\section{HOXB13 and prostate cancer}

Linkage studies previously mapped a prostate cancer susceptibility locus to a $\mathbf{1 5 . 5}-\mathrm{Mb}$ region on chromosome 17. Kathleen Cooney and colleagues now show that a rare, nonsynonymous variant in HOXB13 located in the linkage interval is associated with substantially elevated risk of the disease (N. Engl. J. Med. 366, 141-149, 2012). The authors sequenced 202 genes from the linkage region in 94 families with prostate cancer and identified 4 families carrying the same nonsynonymous variant in HOXB13. Follow-up studies in 5,083 prostate cancer cases and 1,401 controls of European ancestry showed that this variant was enriched in cases (1.4\%) compared to controls $(0.1 \%)$ and was associated with a 20 -fold higher risk of prostate cancer $\left(P=8.5 \times 10^{-7}\right)$. The authors found additional nonsynonymous HOXB13 variants in two other families with prostate cancer and in two prostate cancer cell lines. $H O X B 13$ is expressed in the developing and adult prostate and has previously been implicated in prostate cancer biology. The disease-associated variants map to conserved domains important for protein-protein interactions and DNA-binding activity and may increase prostate cancer risk through a gain-of-function mechanism. 